Draft VERSION OCTOBER 6, 2018

Preprint typeset using $\mathrm{LT}_{\mathrm{E}} \mathrm{X}$ style emulateapj v. 5/2/11

\title{
MASS-DEPENDENT BARYON ACOUSTIC OSCILLATION SIGNAL AND HALO BIAS
}

\author{
QIAO WANG AND Hu ZHAN \\ Key Laboratory of Optical Astronomy, National Astronomical Observatories, Chinese Academy of Sciences, Beijing 100012, China \\ Draft version October 6, 2018
}

\begin{abstract}
We characterize the baryon acoustic oscillations (BAO) feature in halo two-point statistics using $N$-body simulations. We find that nonlinear damping of the BAO signal is less severe for halos in the mass range we investigate than for dark matter. The amount of damping depends weakly on the halo mass. The correlation functions show a mass-dependent drop of the halo clustering bias below roughly $90 \mathrm{~h}^{-1} \mathrm{Mpc}$, which coincides with the scale of the BAO trough. The drop of bias is $4 \%$ for halos with mass $M>10^{14} h^{-1} \mathrm{M}_{\odot}$ and reduces to roughly $2 \%$ for halos with mass $M>10^{13} h^{-1} \mathrm{M}_{\odot}$. In contrast, halo biases in simulations without BAO change more smoothly around $90 h^{-1} \mathrm{Mpc}$. In Fourier space, the bias of $M>10^{14} h^{-1} \mathrm{M}_{\odot}$ halos decreases smoothly by $11 \%$ from wavenumber $k=0.012 h \mathrm{Mpc}^{-1}$ to $0.2 h \mathrm{Mpc}^{-1}$, whereas that of $M>10^{13} h^{-1} \mathrm{M}_{\odot}$ halos decreases by less than $4 \%$ over the same range. By comparing the halo biases in pairs of otherwise identical simulations, one with and the other without BAO, we also observe a modulation of the halo bias. These results suggest that precise calibrations of the mass-dependent BAO signal and scale-dependent bias on large scales would be needed for interpreting precise measurements of the two-point statistics of clusters or massive galaxies in the future.
\end{abstract}

Subject headings: Cosmology: theory — large-scale structure of Universe — dark matter

\section{INTRODUCTION}

Baryon Acoustic Oscillations (BAO, Peebles \& Yu 1970; Sunvaev \& Zeldovich 1970) produce an imprint on the matter distribution by the perturbations in the photon-baryon fluid before recombination. It provides a standard ruler for probing dark energy (e.g., Eisenstein \& Hu 1998; Cooray et al. 2001; Blake \& Glazebrook 2003; Hu \& Haiman 2003; Linder 2003; Seo \& Eisenstein 2003), and is considered to be least affected by systematics (Albrecht et al. 2006). Thus far, the BAO signal has been detected in samples from Sloan Digital Sky Survey (Eisenstein et al. 2005; Padmanabhan et al. 2007; Percival et al. 2010), $2 \mathrm{dF}$ Galaxy Redshift Survey (Cole et al. 2005), 6dF Galaxy Survey (Beutler et al. 2011), WiggleZ Dark Energy Survey (Blake et al. 2011), and Baryon Oscillation Spectroscopic Survey (Anderson et al. 2012). Future spectroscopic and imaging surveys, such as BigBOSS, Euclid, and Large Synoptic Survey Telescope, all aim to determine cosmic distances to sub-percent level precision with the BAO technique (e.g., Zhan et al. 2009; Schlegel et al. 2011).

Although the BAO signal does not evolve in linear theory, nonlinearity causes a slight shift of its scale and significant damping of its amplitude (e.g., Seo et al. 2008, 2010; Sherwin \& Zaldarriaga 2012; McCullagh et al. 2013). These effects must be modeled or corrected in BAO analyses. Given the unprecedented statistical power of future surveys, it is necessary to calibrate the nonlinear BAO signal accurately. Efforts have been made for dark matter with both $N$-body simulations (Smith et al. 2008; Padmanabhan \& White 2009) and perturbation theory (Jeong \& Komatsu 2006; Crocce \& Scoccimarro 2008; Matsubara 2008).

It is reasonable to assume that the halo bias is con-

qwang@nao.cas.cn stant on large scales according to the "peak-background split" or "local bias" model(e.g., Frv \& Gaztanaga 1993; Mo \& White 1996; Scherrer \& Weinberg 1998). For precision cosmology, however, one should quantify the scales of validity as well as any departure from constancy for halos of different masses. Investigations of the halo BAO signal have given hints for scale-dependence in the halo bias even at the BAO scale (e.g., Seo \& Eisenstein 2005; Noh et al. 2009; Desiacques et al. 2010). In this work, we determine the halo correlation functions and power spectra with high precision and clearly demonstrate the mass-dependent halo BAO signal and behavior of the halo bias beyond the BAO scale.

\section{SIMULATIONS}

We run two sets of $N$-body simulations to study the halo BAO signal and halo bias at redshift $z=0$. The initial matter power spectrum of the first set is calculated using CAMB (Lewis, Challinor, \& Lasenby 2000) with the parameters: $\Omega_{\Lambda}=0.73, \Omega_{\mathrm{M}}=0.27, h=0.71, \sigma_{8}=0.8$, $n_{s}=1$, and $\Omega_{\mathrm{b}}=0.045$. That of the second set is calculated using the fitting formula in Eisenstein \& Hu (1998) with the same parameters but no BAO wiggles. For convenience, we refer to them as the BAO simulations and the no-BAO simulations, respectively.

The simulation box is $1 h^{-1} \mathrm{Gpc}$ on each side. With $N_{\mathrm{p}}=640^{3}$ particles, the mass resolution is $2.86 \times$ $10^{11} h^{-1} \mathrm{M}_{\odot}$, and the softening length is set to $50 h^{-1} \mathrm{kpc}$. The simulations are run with GADGET-2 (Springel 2005) from initial redshift $z_{i}=100$ to $z=0$.

There are 150 realizations in each set of the simulations. The initial conditions are generated using 2LPTic (Crocce et al. 2006) with the same random seed for a pair of simulations, one in each set. In this way, the effect of sample variance can be reduced in the comparison between the results from the two sets of simulations.

Halos are identified using the Friends-of-Friends group 


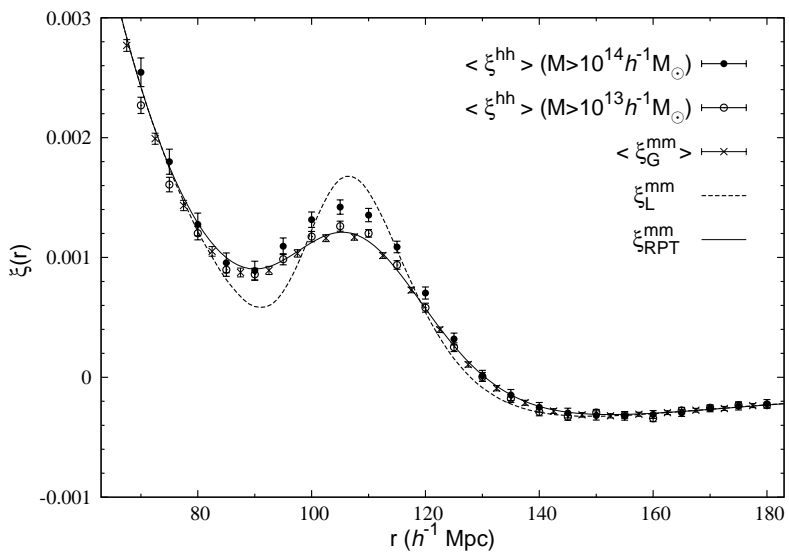

Figure 1. Mass-dependent halo BAO signal at $z=0$. The symbols represent the correlation functions of $M>10^{14} h^{-1} \mathrm{M}_{\odot}$ halos (filled circles), $M>10^{13} h^{-1} \mathrm{M}_{\odot}$ halos (open circles), and dark matter (crosses) in the BAO simulations. The error bars show 1- $\sigma$ errors of the mean correlation functions of 150 realizations. The dashed line is the linear dark matter correlation function, and the solid line is the nonlinear dark matter correlation function from the RPT fitting formula. These correlation functions are normalized in the range of $160-180 h^{-1} \mathrm{Mpc}$.

finder from University of Washington's $N$-Body Shop?. The linking length is set to 0.2 times the mean particle separation. Given the mass resolution, we limit our study to halos more massive than $10^{13} h^{-1} \mathrm{M}_{\odot}$, which corresponds to about 35 simulation particles. The obtained comoving halo number density at $z=0$ is $4.8 \times 10^{-4} h^{3} \mathrm{Mpc}^{-3}$ for $M>10^{13} h^{-1} \mathrm{M}_{\odot}$ and $2.5 \times$ $10^{-5} h^{3} \mathrm{Mpc}^{-3}$ for $M>10^{14} h^{-1} \mathrm{M}_{\odot}$.

\section{HALO BAO AND HALO BIAS}

\subsection{BAO in Halo Correlation Functions}

We calculate the halo two-point correlation functions $\xi^{\text {hh }}(r)$ with the Landy-Szalay estimator (Landy \& Szalay 1993)

$$
\xi^{\mathrm{hh}}(r)=\frac{N_{\mathrm{r}}^{2}}{R R(r)}\left[\frac{H H(r)}{N_{\mathrm{h}}^{2}}-\frac{2 H R(r)}{N_{\mathrm{h}} N_{\mathrm{r}}}+\frac{R R(r)}{N_{\mathrm{r}}^{2}}\right],
$$

where $N_{\mathrm{r}}$ is the size of a uniform random sample, $N_{\mathrm{h}}$ is the size of the halo sample, $H H$ is the number of halo pairs with a separation between $r-\Delta r / 2$ and $r+\Delta r / 2$, $H R$ is the number of pairs between the halo sample and the uniform random sample, and $R R$ is that within the uniform random sample. Since the halo sample from the simulations has periodic boundaries and a uniform selection function, Equation (11) reduces, for an infinitely dense random sample, to

$$
\xi^{\mathrm{hh}}(r)=\frac{H H(r)}{N_{\mathrm{h}}^{2}} \frac{V_{\mathrm{box}}}{V_{\mathrm{bin}}(r)}-1,
$$

where $V_{\text {box }}$ is the volume of the simulation box, and $V_{\text {bin }}(r)$ is the volume of the spherical shell between $r-\Delta r / 2$ and $r+\Delta r / 2$.

The correlation functions $\left\langle\xi^{\text {hh }}\right\rangle$ averaged over the ensemble of the $150 \mathrm{BAO}$ simulations are shown in Figure 1 for halos of mass $M>10^{13} h^{-1} \mathrm{M}_{\odot}$ (open circles) and $M>10^{14} h^{-1} \mathrm{M}_{\odot}$ (filled circles). For com-

\footnotetext{
${ }^{1}$ http://www-hpcc.astro.washington.edu/
}

parison, we also plot the nonlinear dark matter correlation function from the simulations, i.e., the correlation of all simulation particles $\left(\left\langle\xi_{\mathrm{G}}^{\mathrm{mm}}\right\rangle\right.$, crosses $)$, the linear dark matter correlation function $\left(\xi_{\mathrm{L}}^{\mathrm{mm}}\right.$, dashed line), and the nonlinear dark matter correlation function from a convolution of $\xi_{\mathrm{L}}^{\mathrm{mm}}$ with the Gaussian damping factor predicted by Renormalized Perturbation Theory (RPT, Crocce \& Scoccimarro 2006, 2008) ( $\xi_{\mathrm{RPT}}^{\mathrm{mm}}$, solid line). Because it is time consuming to estimate the dark matter correlation function from $640^{3}$ particles with the paircounting method, we assign the particles onto a density grid (hence the subscript G) of $200^{3}$ cells with the cloudin-cell assignment function (Hockney \& Eastwood 1981) and then calculate the correlation function from pairs of the cells.

Studies have shown that nonlinear evolution damps the BAO feature (e.g., Eisenstein et al. 2007; Smith et al. 2008; Seo et al. 2008). Such an effect on dark matter can be fairly accurately modeled with the RPT formulism (see, e.g., Figure 11). Comparing the correlation functions from the simulations, we find that the damping effect of the BAO signal depends on the tracer's mass, which cannot be scaled away by a constant clustering bias. As explained in Eisenstein et al. (2007), the BAO damping is due to the motions of matter or tracers relative to their initial separation, i.e., the Lagrangian displacements between pairs. The mass-dependent damping of the halo $\mathrm{BAO}$ signal suggests that the characteristics of halo motions are also mass-dependent. For a test, we measure the pairwise velocity dispersion $\sigma_{\mathrm{pv}}$, which is proportional to the rms Lagrangian displacement in the Zel'dovich approximation, and find that $\sigma_{\mathrm{pv}}=650.1 \pm 6.0 \mathrm{~km} \mathrm{~s}^{-1}$ for $M>10^{13} h^{-1} \mathrm{M}_{\odot}$ halos and $\sigma_{\mathrm{pv}}=632.5 \pm 8.4 \mathrm{~km} \mathrm{~s}^{-1}$ for $M>10^{14} h^{-1} \mathrm{M}_{\odot}$ halos over the separations of 90 $120 h^{-1} \mathrm{Mpc}$. These results appear to be consistent with Figure 1in the sense that larger pairwise random motions would damp the BAO signal more. However, we note that nonlinear evolution weakens the correspondence betweeen the pairwise velocity and the Lagrangian displacement. A more thorough investigation is needed to fully understand the mass-dependent BAO damping.

\subsection{Halo Bias from Correlation Functions}

The difference between the halo correlation functions in Figure 1 can be accommodated with scale-dependent biases. We estimate the halo bias in two ways:

$$
\begin{aligned}
b_{\mathrm{CC}}(r) & \equiv\left\langle\xi^{\mathrm{hh}}(r) / \xi^{\mathrm{hm}}(r)\right\rangle, \\
b_{\mathrm{G}}(r) & \equiv\left\langle\xi_{\mathrm{G}}^{\mathrm{hh}}(r)\right\rangle^{\frac{1}{2}} /\left\langle\xi_{\mathrm{G}}^{\mathrm{mm}}(r)\right\rangle^{\frac{1}{2}},
\end{aligned}
$$

where $\xi^{\mathrm{hm}}$ is the cross correlation between the halos and dark matter in each simulation, and $\xi_{\mathrm{G}}^{\mathrm{hh}}$ is the correlation function calculated from the halo density grid (analogous to $\left.\xi_{\mathrm{G}}^{\mathrm{mm}}\right)$. The cross correlation is given by

$$
\xi^{\mathrm{hm}}(r)=\frac{H M(r)}{N_{\mathrm{h}} N_{\mathrm{p}}} \frac{V_{\mathrm{box}}}{V_{\mathrm{bin}}(r)}-1,
$$

where $H M$ is the number of halo-simulation particle pairs with a separation between $r-\Delta r / 2$ and $r+\Delta r / 2$. One might attempt to take an ensemble average of the ratio $\xi_{\mathrm{G}}^{\mathrm{hh}} / \xi_{\mathrm{G}}^{\mathrm{mm}}$ as an estimator for $b^{2}$. Such a ratio can fluctuate wildly and even be negative in some realizations when the correlations are close to zero. Equation (4) is 


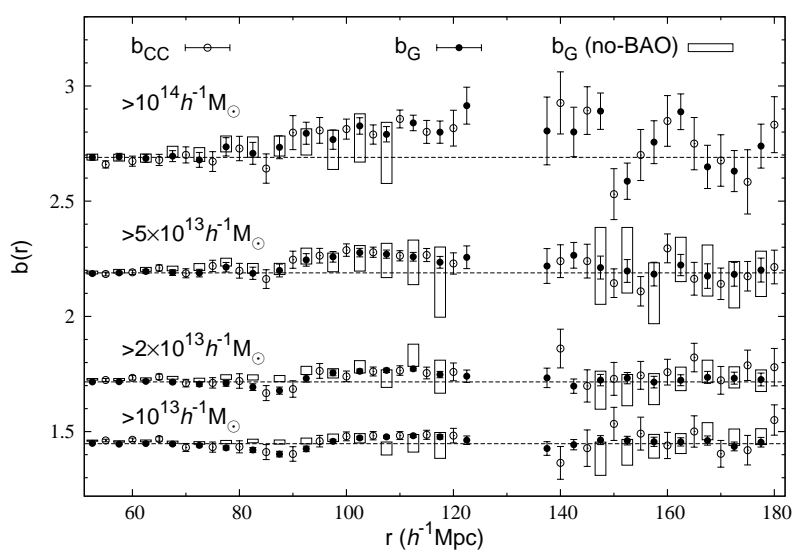

Figure 2. Halo bias from the correlation functions. Open circles and filled circles represent, respectively, $b_{\mathrm{CC}}$ and $b_{\mathrm{G}}$ (see Equations 3 and (4) in the BAO simulations. Horizontal lines mark the average values of $b_{\mathrm{G}}$ between $50 h^{-1} \mathrm{Mpc}$ and $70 h^{-1} \mathrm{Mpc}$. Boxes represent $b_{\mathrm{G}}$ in the no-BAO simulations.

better behaved, though the covariance between $\xi_{\mathrm{G}}^{\mathrm{hh}}$ and $\xi_{\mathrm{G}}^{\mathrm{mm}}$ needs to be accounted for when determining the uncertainties of $b_{\mathrm{G}}$.

Figure 2 shows the behavior of the halo bias for four mass ranges as labeled. Since the halo mass function is fairly steep at $M>10^{13} h^{-1} \mathrm{M}_{\odot}$, each mass range is dominated by halos at its low-mass end. The halo bias cannot be determined accurately when the correlation functions are close to zero. Therefore, we leave a gap where the errors become too large to be informative.

For the BAO simulations, the results of the two different estimators, $b_{\mathrm{CC}}$ (open circles) and $b_{\mathrm{G}}$ (filled circles), are consistent with each other over the scales and masses of interest. They both show a systematic offset between the average halo bias over $50-70 h^{-1} \mathrm{Mpc}$ (dashed lines) and that over the BAO peak (roughly 95$115 h^{-1} \mathrm{Mpc}$ ) for all the four mass ranges. The decrement of the bias below the transition scale depends on the halo mass: $4 \%$ for $M>10^{14} h^{-1} \mathrm{M}_{\odot}$ and roughly $2 \%$ for $M>10^{13} h^{-1} \mathrm{M}_{\odot}$. This finding is relevant to BAO measurements from luminous red galaxies, which are mostly central galaxies in halos of masses from a few times $10^{13} h^{-1} \mathrm{M}_{\odot}$ to $10^{14} h^{-1} \mathrm{M}_{\odot}$ (Zheng et al. 2009).

To see whether it is a coincidence that the bias drops near the trough of the BAO signal, we contrast the results from the BAO simulations (circles) with those from the no-BAO simulations (boxes) in Figure 2. Since the latter changes gradually around $90 h^{-1} \mathrm{Mpc}$, we conclude that the drop of the halo bias in the BAO simulations is associated with the presence of BAO.

The scale dependence of the halo bias around the BAO scale has been studied previously. For example, one may find a hint for a bump of the halo bias around $95 h^{-1} \mathrm{Mpc}$ for halos with mass $M \gtrsim$ $10^{14} h^{-1} \mathrm{M}_{\odot}$ in Manera, Sheth. \& Scoccimarro (2010) and Manera \& Gaztañaga (2011). A peak of the halo bias $\left(M>1.2 \times 10^{14} h^{-1} \mathrm{M}_{\odot}\right)$ near $100 h^{-1} \mathrm{Mpc}$ is also detected in Desjacques et al. (2010).

This work improves the statistics of the halo bias around the BAO scale. The mass-dependent drop of the bias below $90 h^{-1} \mathrm{Mpc}$ is well detected for halo masses down to $10^{13} h^{-1} \mathrm{M}_{\odot}$. Our results are consistent with a flat bias over $95-115 h^{-1} \mathrm{Mpc}$. We also extend the scale

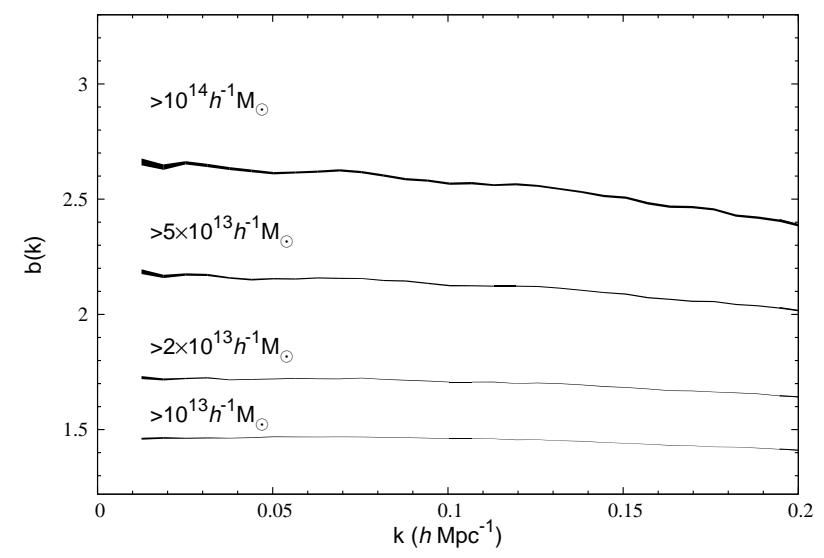

Figure 3. Halo bias from the power spectra. The width of each curve represents 1- $\sigma$ uncertainties of the mean bias of the ensemble.

of interest up to $180 h^{-1} \mathrm{Mpc}$ where halos are anticorrelated (so is dark matter). Because the correlation functions are close to zero in the range of $140-180 h^{-1} \mathrm{Mpc}$, the uncertainties of the halo bias in this range are considerably larger than those below $120 h^{-1} \mathrm{Mpc}$. Nevertheless, the results are consistent with a flat bias over $140-180 h^{-1} \mathrm{Mpc}$.

\subsection{Halo Bias from Power Spectra}

One can also estimate the halo bias from the halo power spectrum $P^{\mathrm{hh}}(k)$ and the dark matter power spectrum $P^{\mathrm{mm}}(k)$ with

$$
b_{\mathrm{PS}}(k) \equiv\left\langle\left[P^{\mathrm{hh}}(k) / P^{\mathrm{mm}}(k)\right]^{\frac{1}{2}}\right\rangle .
$$

To obtain the power spectra, we assign the halos and simulation particles to density grids of $512^{3}$ cells using the Daubechies D12 wavelet scaling function, which has superior performance for measuring the power spectrum (Cui et al. 2008). We then take $P^{\mathrm{hh}}(k)=\left\langle\left|\hat{\delta}^{\mathrm{h}}(\mathbf{k})\right|_{|\mathbf{k}|=k}^{2}\right\rangle_{\mathrm{G}}-V_{\mathrm{box}} N_{\mathrm{h}}^{-1}$ and $P^{\mathrm{mm}}(k)=$ $\left\langle\left|\hat{\delta}^{\mathrm{m}}(\mathbf{k})\right|_{|\mathbf{k}|=k}^{2}\right\rangle_{\mathrm{G}}-V_{\text {box }} N_{\mathrm{p}}^{-1}$, where $\hat{\delta}^{\mathrm{h}}(\mathbf{k})$ and $\hat{\delta}^{\mathrm{m}}(\mathbf{k})$ are the discrete Fourier modes of the halo grid and the dark matter grid, respectively, and $\langle\ldots\rangle_{\mathrm{G}}$ denotes an average within each grid.

Figure 3 presents the halo bias determined from the power spectra for the same mass ranges as in Figure 2. Although one does not expect the halo bias to be absolutely constant on large scales, it is still surprising that the bias of $M>10^{14} h^{-1} \mathrm{M}_{\odot}$ halos falls by $11 \%$ from $k=0.012 h \mathrm{Mpc}^{-1}$ to $k=0.2 h \mathrm{Mpc}^{-1}$. For halos of mass $M>10^{13} h^{-1} \mathrm{M}_{\odot}$, the bias falls by less than $4 \%$ over the same scales, consistent with the result of $M>1.11 \times 10^{13} h^{-1} \mathrm{M}_{\odot}$ in Pollack, Smith, \& Porciani (2012).

One may notice that there are slight oscillations in the halo bias, which are more pronounced for more massive halos. This behavior is discussed in the next subsection.

\subsection{Damping of BAO and Bias Modulation}

We use the no-BAO simulations as references to examine the damping of the BAO signal in Fourier space. The halo and dark matter power spectra from each simulation with $\mathrm{BAO}$ are divided, respectively, by those from 


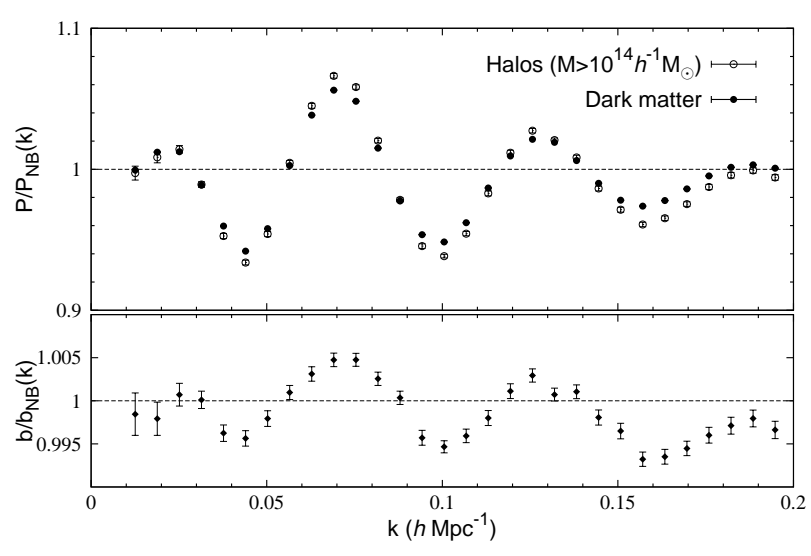

Figure 4. Upper panel: Ratio of the power spectrum from the $\mathrm{BAO}$ simulations to that from the no-BAO simulations. Filled circles represent dark matter, and open circles represent $M>$ $10^{14} h^{-1} \mathrm{M}_{\odot}$ halos. Lower panel: Ratio of the halo bias in the BAO simulations to that in no-BAO simulations for $M>10^{14} h^{-1} \mathrm{M}_{\odot}$ halos.

the corresponding no-BAO simulation. Because the pair of simulations share the same random seed for their initial conditions, the sample variance is greatly reduced. The power spectrum ratios are shown in the upper panel of Figure 4 for $M>10^{14} h^{-1} \mathrm{M}_{\odot}$ halos $\left(P^{\mathrm{hh}} / P_{\mathrm{NB}}\right.$, open circles) and dark matter ( $P^{\mathrm{mm}} / P_{\mathrm{NB}}^{\mathrm{mm}}$, filled circles $)$. One can see that the BAO signal in the massive halo power spectrum suffers less damping than that in the dark matter power spectrum.

There is a subtle difference between Figure 1 and Figure 4. Figure 11llustrates a mismatch between the shape of the halo correlation functions and that of dark matter around the $\mathrm{BAO}$ scale. It does not associate the very presence of BAO with the mismatch. The latter could be an intrinsic difference between the halos and dark matter that happens to occur around the BAO scale. Figure 3 establishes such a link, but the uncertainty is a little high for $M>10^{14} h^{-1} \mathrm{M}_{\odot}$ halos. The power spectrum ratios in Figure 4 suppress the intrinsic difference between halos and dark matter and reduce uncertainties arising from the sample variance. It demonstrates for $M>10^{14} h^{-1} \mathrm{M}_{\odot}$ halos that the difference between the halo and dark matter BAO signals is truly due to the difference in the BAO damping mechanism.

By definition, the above effect is attributed to the halo bias. The lower panel of Figure 4 shows the ratio of the halo bias in the BAO simulations to that in no-BAO simulations for $M>10^{14} h^{-1} \mathrm{M}_{\odot}$ halos (same as the halo data divided by the dark matter data in the upper panel). The result oscillates in phase with $\mathrm{BAO}$ with an amplitude of $0.5 \%$, suggesting a modulation of the halo bias by BAO. This would not be sensible if halos distributed exactly in proportion to dark matter. However, if there is nonlinearity in the halo bias, as already evident from its scale dependence in Figures 2 and 3 , the bias will depend on the shape of the dark matter power spectrum to some extent.

\section{DISCUSSION}

We have demonstrated with high significance that the $\mathrm{BAO}$ signal in the halo distribution is dependent on the halo mass. In the correlation function, the relative amplitude of the BAO peak of $M>10^{14} h^{-1} \mathrm{M}_{\odot}$ halos is considerably higher than that of $M>10^{13} h^{-1} \mathrm{M}_{\odot}$ halos, which in turn is higher than that of dark matter. Since galaxies reside in halos, the BAO signal in the galaxy distribution must also depend on the mass of the host halos.

We further show that the halo bias is scale-dependent even on very large scales. This could be a significant source of systematic errors when one extracts cosmological information (e.g., neutrino masses, Lahav et al. 2010), from the overall shape of the galaxy power spectrum.

Recently, Hong et al. (2012) detect a higher-thanexpected $\mathrm{BAO}$ peak in the correlation function of galaxy clusters $\left(M \gtrsim 10^{14} h^{-1} \mathrm{M}_{\odot}\right)$. Since they fix the baryon fraction while fitting other parameters, the excess BAO signal has to be compensated by a very low matter fraction. Although the difference between the halo and dark matter BAO signals in our study is not sufficient to explain all the excess BAO signal in Hong et al. (2012), it should be nonetheless a significant contributing factor.

One might expect the trend with halo mass in Figures 2 and 3 to continue below $10^{13} h^{-1} \mathrm{M}_{\odot}$. Investigation with $M>1.37 \times 10^{12} h^{-1} \mathrm{M}_{\odot}$ halos (Sato \& Matsubara 2011) shows that the bias of such halos at $z \geq 0.5$ varies significantly with scales. A simple extrapolation of their results to $z=0$ suggests that the fractional variation of the bias of $M>1.37 \times 10^{12} h^{-1} \mathrm{M}_{\odot}$ halos could be even larger than that of $M>10^{13} h^{-1} \mathrm{M}_{\odot}$ halos. Therefore, it is prudent not to extrapolate our results to lower masses.

Besides damping the BAO signal, nonlinear evolution also shifts the acoustic scale slightly. We estimate this effect using the fitting formulae in Seo et al. (2008) and find that the fractional shift of the BAO feature toward higher wavenumber is $(0.28 \pm 0.16) \%,(0.47 \pm 0.20) \%$, $(0.54 \pm 0.28) \%$, and $(0.62 \pm 0.23) \%$ for dark matter, $M>10^{13} h^{-1} \mathrm{M}_{\odot}$ halos, $M>2 \times 10^{13} h^{-1} \mathrm{M}_{\odot}$ halos, and $M>5 \times 10^{13} h^{-1} \mathrm{M}_{\odot}$ halos, respectively. These results are consistent with those in Seo et al. (2010) and Sherwin \& Zaldarriaga (2012). The simple fitting does not converge well for the highest mass range in our study. In this case, one can use the more sophisticated fitting formula for halos proposed in Padmanabhan \& White (2009).

Future galaxy surveys could probe effective volumes of a few hundred $\mathrm{Gpc}^{3}$, similar to the total volume of the simulations in this study. The effect of mass-dependent halo BAO signal and the scale-dependent halo bias on large scales could be easily detected in the galaxy statistics. In other words, it will be necessary to accurately calibrate these effects for precision cosmology in the future

This work was supported by the National Natural Science foundation of China grant No. 11033005, the National Key Basic Research Science Foundation of China grant No. 2010CB833000, and the Bairen program from the Chinese Academy of Sciences.

\section{REFERENCES}

Albrecht, A., Bernstein, G., Cahn, R., et al. 2006, ArXiv Astrophysics e-prints

Anderson, L., Aubourg, E., Bailey, S., et al. 2012, ArXiv e-prints 
Beutler, F., Blake, C., Colless, M., et al. 2011, MNRAS, 416, 3017 Blake, C., \& Glazebrook, K. 2003, ApJ, 594, 665

Blake, C., Davis, T., Poole, G. B., et al. 2011, MNRAS, 415, 2892 Cole, S., Percival, W. J., Peacock, J. A., et al. 2005, MNRAS, 362,505

Cooray, A., Hu, W., Huterer, D., \& Joffre, M. 2001, ApJ, 557, L7

Crocce, M., Pueblas, S., \& Scoccimarro, R. 2006, MNRAS, 373, 369

Crocce, M., \& Scoccimarro, R. 2006, Phys. Rev. D, 73, 063520

—. 2008, Phys. Rev. D, 77, 023533

Cui, W., Liu, L., Yang, X., et al. 2008, ApJ, 687, 738

Desjacques, V., Crocce, M., Scoccimarro, R., \& Sheth, R. K. 2010, Phys. Rev. D, 82, 103529

Eisenstein, D. J., \& Hu, W. 1998, ApJ, 496, 605

Eisenstein, D. J., Seo, H.-J., \& White, M. 2007, ApJ, 664, 660

Eisenstein, D. J., Zehavi, I., Hogg, D. W., et al. 2005, ApJ, 633, 560

Fry, J. N., \& Gaztanaga, E. 1993, ApJ, 413, 447

Hockney, R. W., \& Eastwood, J. W. 1981, Computer Simulation Using Particles

Hong, T., Han, J. L., Wen, Z. L., Sun, L., \& Zhan, H. 2012, ApJ, 749,81

Hu, W., \& Haiman, Z. 2003, Phys. Rev. D, 68, 063004

Jeong, D., \& Komatsu, E. 2006, ApJ, 651, 619

Lahav, O., Kiakotou, A., Abdalla, F. B., \& Blake, C. 2010, MNRAS, 405, 168

Landy, S. D., \& Szalay, A. S. 1993, ApJ, 412, 64

Lewis, A., Challinor, A., \& Lasenby, A. 2000, ApJ, 538, 473

Linder, E. V. 2003, Phys. Rev. D, 68, 083504

Manera, M., \& Gaztañaga, E. 2011, MNRAS, 415, 383
Manera, M., Sheth, R. K., \& Scoccimarro, R. 2010, MNRAS, 402, 589

Matsubara, T. 2008, Phys. Rev. D, 78, 083519

McCullagh, N., Neyrinck, M. C., Szapudi, I., \& Szalay, A. S.

2013, ApJ, 763, L14

Mo, H. J., \& White, S. D. M. 1996, MNRAS, 282, 347

Noh, Y., White, M., \& Padmanabhan, N. 2009, Phys. Rev. D, 80, 123501

Padmanabhan, N., \& White, M. 2009, Phys. Rev. D, 80, 063508

Padmanabhan, N., Schlegel, D. J., Seljak, U., et al. 2007, MNRAS, 378, 852

Peebles, P. J. E., \& Yu, J. T. 1970, ApJ, 162, 815

Percival, W. J., Reid, B. A., Eisenstein, D. J., et al. 2010, MNRAS, 401, 2148

Pollack, J. E., Smith, R. E., \& Porciani, C. 2012, MNRAS, 420, 3469

Sato, M., \& Matsubara, T. 2011, Phys. Rev. D, 84, 043501

Scherrer, R. J., \& Weinberg, D. H. 1998, ApJ, 504, 607

Schlegel, D., Abdalla, F., Abraham, T., et al. 2011, ArXiv e-prints

Seo, H.-J., \& Eisenstein, D. J. 2003, ApJ, 598, 720

—. 2005, ApJ, 633, 575

Seo, H.-J., Siegel, E. R., Eisenstein, D. J., \& White, M. 2008, ApJ, 686, 13

Seo, H.-J., Eckel, J., Eisenstein, D. J., et al. 2010, ApJ, 720, 1650

Sherwin, B. D., \& Zaldarriaga, M. 2012, Phys. Rev. D, 85, 103523

Smith, R. E., Scoccimarro, R., \& Sheth, R. K. 2008,

Phys. Rev. D, 77, 043525

Springel, V. 2005, MNRAS, 364, 1105

Sunyaev, R. A., \& Zeldovich, Y. B. 1970, Ap\&SS, 7, 3

Zhan, H., Knox, L., \& Tyson, J. A. 2009, ApJ, 690, 923

Zheng, Z., Zehavi, I., Eisenstein, D. J., Weinberg, D. H., \& Jing,

Y. P. 2009, ApJ, 707, 554 